\title{
EDITORIAL
}

\section{Are angiotensin II receptor blockers indicated in chronic heart failure?}

\section{Komajda}

Have the theoretical advantages of the angiotensin II receptor blockers become reality for the treatment of chronic heart failure?
Correspondence to: Professor Michel Komajda, Service de Cardiologie, Hôpital Pitié-Salpétrière 47-83 Boulevard de I'hôpital, 75651 Paris Cedex 13, France; michel.komajda@ psl.ap-hop-paris.fr
C hronic heart failure is one of the most serious cardiac problems encountered in clinical practice. Modulation of the renin angiotensin system is a key element in the treatment of this syndrome. There is overwhelming evidence in favour of the benefit of angiotensin converting enzyme (ACE) inhibitors on morbidity and mortality in mild to severe heart failure and in heart failure or left ventricular dysfunction following acute myocardial infarction. ${ }^{1}$ It is not known, however, whether the benefit of ACE inhibition is solely attributable to blockade of angiotensin II production or also related to bradykinin accumulation. ${ }^{2}$ Moreover, bradykinin accumulation has been implicated in the adverse effects observed with ACE inhibitors such a cough and might result in prejunctional noradrenaline (norepinephrine) release. ${ }^{3}$

The development of orally active, non-peptide angiotensin II type I receptor blockers (ARBs) has raised hopes for a new generation of modulators of the renin angiotensin system better tolerated and potentially more powerful. Since ARBs block directly the binding of angiotensin II to the receptor which mediates harmful biological actions such as vasoconstriction, aldosterone synthesis, and trophic effects, one potential advantage is to avoid the ACE independent pathway generation of angiotensin II.

Have these theoretical advantages become reality for the treatment of chronic heart failure?

The excitement in designing a large trial comparing an ARB to an ACE inhibitor in chronic heart failure is mainly based on the results of the ELITE I trial. ${ }^{4}$ In this study, which enrolled 722 elderly patients and compared losartan $50 \mathrm{mg} /$ day to captopril $150 \mathrm{mg} /$ day, it was shown that a secondary end point favoured losartan with a risk reduction of death/hospital admission for heart failure of $32 \%$, primarily due to a decrease in mortality. However, it was argued that this was only a secondary end point (the primary objective was persisting increase in serum creatinine) in a trial which did not meet the standard criteria of size and duration for licensing in chronic heart failure, and that the confidence interval for the point estimate of the risk reduction was wide.

Nevertheless, based on these results, a large morbidity and mortality trial was undertaken, ELITE II, which enrolled 3152 patients over 60 years randomised to losartan $50 \mathrm{mg}$ or captopril titrated to $150 \mathrm{mg}$ daily. The trial was based on the assumption that losartan would be able to demonstrate a $25 \%$ decrease in mortality over captopril. Unfortunately, ELITE II failed to demonstrate such a superiority and there was no difference between the two treatment groups regarding all cause mortality, sudden death or resuscitated arrests. ${ }^{5}$ The only significant difference observed was the lower incidence of adverse effects leading to discontinuation of the study in the losartan group. This led the investigators to conclude that "it still remains to be established whether angiotensin II antagonists are a fully effective substitute for ACE inhibitors in heart failure" and state that ARBs might be a useful alternative agent to block the renin angiotensin system in patients who do not tolerate ACE inhibitors. Interestingly, a subgroup analysis of patients treated with/without ( $22 \% / 78 \%$ of the population) $\beta$ blockers suggested possible interaction, although overlapping confidence intervals for these two subgroups suggested a greater benefit with the $\beta$ blocker-ACE inhibitor combination.

\section{Val-HeFT TRIAL}

A second large trial, Val-HeFT, was presented at the Scientific Sessions of the American Heart Association in 2000. Val-HeFT enrolled 5010 patients, mainly New York Heart Association (NYHA) functional class II and III with low ejection fraction and evidence of left ventricular dilatation.

Patients were randomised to valsartan titrated up to $160 \mathrm{mg}$ twice daily or placebo on a background treatment of ACE inhibitors (93\%), diuretics $(86 \%), \beta$ blockers $(36 \%)$, or digitalis $(67 \%)$. There were two primary end points: all cause mortality alone or combined with resuscitated death, hospitalisation for heart failure or need for therapeutic doses of intravenous inotropes or vasodilators for at least four hours. With an average follow up of two years, Val-HeFT did not demonstrate any difference in all cause mortality between the two arms but showed a significant $13 \%$ reduction in the occurrence of the composite primary end point favouring the combination. The study also demonstrated a significant reduction

Abbreviations: $A C E-I$, angiotensin converting enzyme inhibitor; ARB, angiotensin II receptor blocker; CHARM, candesarton in heart failure-assessment of reduction in mortality and morbidity; ELITE, evaluation of losartan in the elderly; NYHA, New York Heart Association; OPTIMAAL, optimal trial in myocardial infarction with the angiotensin II antagonist losartan; RENAAL, reduction of end points in NIDDM with the angiotensin II antagonist losartan; VALIANT, valsartan in acute myocardial infarction trial; Val-HeFT, valsartan heart failure trial 
$(27 \%)$ in heart failure related hospitalisation, an improvement in NYHA class, and a better quality of life in patients receiving valsartan. The rate of discontinuation for side effects was higher in the valsartan group $(9.9 \%)$ than with placebo $(7.2 \%)$, and the most common reasons for discontinuation were dizziness, hypotension, and renal impairment. Interestingly, the effect of the ARB on the composite primary end point was particularly important in patients not receiving a $\beta$ blocker (64\%), whereas the triple therapy $\beta$ blocker-ACE inhibitor-ARB was not as effective as the ACE inhibitor- $\beta$ blocker combination.

"The results of Val-HeFT demonstrate a modest benefit for ARBs over ACE inhibitors in morbidity and mortality"

As they stand, the results of Val-HeFT demonstrate a modest benefit for ARBs over ACE inhibitors in morbidity and mortality, and this effect is apparently mainly driven by the improvement in morbidity. However, the potential interaction between ARBs and $\beta$ blockers raises two hypotheses: (1) the dual combination ACE inhibitor- $\beta$ blocker is so powerful in reducing morbidity and mortality that the addition of a third neuromodulator, valsartan, does not result in any benefit because of a "plateau" effect ; (2) the reduction of blood pressure observed with the triple therapy ARB-ACE inhibitor- $\beta$ blocker is harmful in this predominantly ischaemic heart failure population. It is therefore important to wait for detailed analysis, including blood pressure variations in patients with/ without $\beta$ blockers.

\section{FUTURE ARB TRIALS}

Several ongoing or planned trials should bring valuable information in the coming years and allow us to identify the indications for ARBs in chronic heart failure. CHARM is a large ongoing trial with candesartan and has three arms: patients intolerant to ACE inhibitor, patients with heart failure with preserved systolic function, and one arm comparing the combination of ACE inhibitor plus ARB to ACE inhibitor alone. CHARM is therefore the only ongoing trial in which an ARB is compared to placebo in ACE intolerant patients. Each of the three arms has a $84-90 \%$ power to detect an $18 \%$ reduction in the combined end point of cardiovascular death or heart failure hospitalisation, and the three components will be pooled to study the effects on all cause mortality.

OPTIMAAL and VALIANT are two large ongoing trials in post-myocardial infarction. OPTIMAAL has enrolled more than 5000 high risk patients post-myocardial infarction with heart failure and depressed ejection fraction and assumes that losartan $50 \mathrm{mg}$ will decrease overall mortality by $20 \%$ versus captopril. VALIANT will enroll 14500 post-myocardial patients with heart failure or left ventricular dysfunction and will compare captopril, valsartan, and their combination on overall mortality. Both post-myocardial infarction trials are powered to test not only the superiority of the ARB but also the non-inferiority. Other trials comparing various dose regimens or focusing on heart failure with preserved systolic function are presently under discussion.

Overall, we can therefore expect information on more than 30000 patients treated with ARBs, either singly or in combination with an ACE inhibitor, for chronic heart failure or following a myocardial infarction in the next five years.

\section{RECOMMENDATIONS FOR USE OF ARBS}

In the mean time, we should adopt a conservative approach and recommend the use of ARBs in chronic heart failure only as second line treatment.

\section{Patients intolerant to ACE inhibitor}

The rationale for using ARBs as a substitute for ACE inhibitors when this treatment is not tolerated is based on several lines of evidence:
- In ELITE II, there was no difference in outcome between losartan and captopril treated groups although this trial was not powered to test equivalence

- In Val-HeFT, patients without ACE inhibitor treatment particularly benefit from use of ARBs

- In the recently presented RENAAL trial, where losartan $100 \mathrm{mg}$ was compared to placebo in 1513 predominantly hypertensive type 2 diabetic patients, a significant reduction in the rate of heart failure hospitalisations was observed in the losartan group.

Taken together, these results suggest that ARBs might be a plausible alternative for modulation of the renin angiotensin system in patients who cannot take ACE inhibitors. However, it is highly desirable to design a specific trial in this population of ACE intolerant patients.

\section{Patients intolerant to $\beta$ blockers}

As mentioned previously, the benefit of the combination ACE inhibitor + ARB was more important in patients not receiving a $\beta$ blocker in the Val-HeFT trial. An implication might therefore be to use ARBs in combination with ACE inhibitors in heart failure patients who cannot otherwise receive a $\beta$ blocker because of contraindications or intolerance.

\section{Patients remaining severely symptomatic despite "optimal" treatment with ACE inhibitor, $\beta$ blockers, diuretic, and spironolactone if tolerated}

The addition of an ARB could be considered in these symptomatic patients to reduce hospitalisation for heart failure and improve symptoms as an alternative to digitalis. However, one should not minimise the difficulties or limitations resulting from the use of this polypharmacy on blood pressure, renal function, and electrolytes in these severely symptomatic patients.

Another issue to be addressed is the dose regimen: it is unclear whether low/moderate doses (ELITE II) or high doses (Val-HeFT) are preferable for the treatment of heart failure. Obviously, we need additional studies comparing the effect of various doses on morbidity, mortality, and safety.

Finally, we do not know at present whether ARBs are beneficial or not in heart failure with preserved systolic function, and we will have to wait for the results of the ongoing CHARM trial or future trials to address this important issue.

Obviously, we are lacking data. The large ongoing trials should generate a wealth of information regarding dosage, indications, interactions, associations, and adverse effects, and help clarify all the pending problems. Meanwhile, we should adopt the cautious approach stated in the updated version of the guidelines for the treatment of heart failure of the European Society of Cardiology. ${ }^{6}$

\section{REFERENCES}

1 Flather MD, Yusuf S, Kober L, et al for the ACE-Inhibitor Myocardial Infarction Collaborative Group. Long-term ACE inhibitor therapy in patients with heart failure or left-ventricular dysfunction: a systematic overview of data from individual patients. Lancet 2000;355:1575-81.

2 Timmermans PBMWM, Wong PC, Chiu AT, et al. Angiotensin II receptors and angiotensin II receptor-antagonists. Pharmacol Rev 1993;45:205-51

3 Rump LC, Oberhauser V, Schwerffeger E, et al. Experimental evidence to support ELITE. Lancet 1998; 351:644-5.

4 Pitt B, Segal R, Martinez FA, et al on behalf of the ElITE study. Randomised trial of losartan versus captopril in patients overt 65 with heart failure. Lancet 1997;349:747-52

5 Pitt B, Poole-Wilson P, Segal R, et al. Effect of losartan compared with captopril on mortality in patients with symptomatic heart failure: randomised trial. The losartan heart failure survival study ELITE II. Lancet 2000;355: 1582-7.

6 European Society of Cardiology. Guidelines for the diagnosis and treatment of chronic heart failure. Task force for the diagnosis and treatment of chronic heart failure of the European Society of Cardiology. Eur Heart J 2001;22:1527-60. 